\title{
Locomotor Recovery in Spinal Cord-Injured Rats Treated with an Antibody Neutralizing the Myelin-Associated Neurite Growth Inhibitor Nogo-A
}

\author{
Doron Merkler, ${ }^{1}$ Gerlinde A. S. Metz, ${ }^{2}$ Olivier Raineteau, ${ }^{1}$ Volker Dietz, ${ }^{3}$ Martin E. Schwab, ${ }^{1}$ and \\ Karim Fouad 1,3 \\ 1Department of Neuromorphology, Brain Research Institute, University and Swiss Federal Institute of Technology Zürich, \\ 8057 Zürich, Switzerland, '2Department of Psychology, University of Lethbridge, Lethbridge, Alberta T1K 3M4, Canada, \\ and ${ }^{3}$ Swiss Paraplegic Centre, University Hospital Balgrist, University of Zürich, 8008 Zürich, Switzerland
}

The limited plastic and regenerative capabilities of axons in the adult mammalian CNS can be enhanced by the application of a monoclonal antibody $(\mathrm{mAb}), \mathrm{IN}-1$, raised against the myelinassociated neurite growth inhibitor Nogo-A. The aim of the present study was to investigate the effects of this treatment on the functional recovery of adult rats with a dorsal overhemisection of the spinal cord. Directly after injury, half of the animals were implanted with $\mathrm{mAb} I \mathrm{~N}$-1-secreting hybridoma cells, whereas the others received cells secreting a control antibody (anti-HRP). A broad spectrum of locomotor tests (open field locomotor) score, grid walk, misstep withdrawal response, narrow-beam crossing) was used to characterize locomotor recovery during the 5 weeks after the injury. In all behavioral tests, the recovery in the mAb IN-1-treated group

Spontaneous regeneration of injured axons or plastic rearrangements of spared fiber systems after spinal cord injury in mammals are very limited. The major reasons for this poor spontaneous repair capacity seem to be the insufficient growth response of neurons to injury, the growth-inhibitory components of the adult CNS tissue, and the formation of cysts and scar tissue at the injury site. Attempts to overcome local barriers by grafting peripheral nerve bridges (Cheng et al., 1996), Schwann cells (Li and Raisman, 1994; Xu et al., 1995), or olfactory ensheathing cells ( $\mathrm{Li}$ et al., 1997; Ramon-Cueto et al., 2000) have led to regenerative fiber growth and in some instances to behavioral recovery in animal models of spinal cord injury, although the mechanistic understanding of this recovery remains incomplete because of the complexity of these interventions; Schwann cells and olfactory ensheathing glia are also important sources of trophic factors and extracellular matrix molecules (Guénard et al., 1993; Franklin and Barnett, 2000). Attempts to increase the neuronal growth

Received Dec. 1, 2000; revised March 5, 2001; accepted March 6, 2001.

This study was supported by the Swiss National Science Foundation (Grant 4038-043918.95), by the Spinal Cord Consortium of the Christopher Reeve Paralysis Foundation (Springfield, NJ), and by the International Institute for Research in Paraplegia (Grant P49/99). We thank H. J. Kasper and J. Scholl for technical support, B. Niederöst for hybridoma cell supply, and Drs. A. McKinney and D. Pinschewer for critically reading this manuscript.

Correspondence should be addressed to Dr. Karim Fouad, Faculty of Rehabilitation Medicine, The University of Alberta, Corbett Hall, Edmonton, Alberta T6G 2G4, Canada. E-mail: Karim.Fouad@ualberta.ca.

Copyright (C) 2001 Society for Neuroscience $0270-6474 / 01 / 213665-09 \$ 15.00 / 0$ was significantly augmented compared with the control antibodytreated rats. EMG recordings of flexor and extensor muscles during treadmill walking confirmed the improvement of the locomotor pattern in the mAb IN-1-treated rats; step-cycle duration, rhythmicity, and coupling of the hindlimbs were significantly improved. No differences between the two groups with regard to nociception were observed in the tail flick test 5 weeks after the operation. These results indicating improved functional recovery suggest that the increased plastic and regenerative capabilities of the CNS after Nogo-A neutralization result in a functionally meaningful rewiring of the motor systems.

Key words: spinal cord injury; functional recovery; locomotion; Nogo-A; regeneration; plasticity; rats response by local applications of neurotrophic factors to spinal cord injury sites have led to increased sprouting of CNS fibers and dorsal root axons (Schnell et al., 1994; Grill et al., 1997; Kobayashi et al., 1997; Liu et al., 1999; Ramer et al., 2000). In addition, neutralization of the myelin-associated neurite growth inhibitor Nogo-A (Chen et al., 2000) with a monoclonal antibody (mAb), IN-1, resulted in growth of lesioned and uninjured CNS fibers (Schnell and Schwab, 1990, 1993; Bregman et al., 1995; Thallmair et al., 1998; Brosamle et al., 2000).

A major question arising from all these studies is whether regenerating or plastically growing neurons can be reconnected in a functionally meaningful way in the adult mammalian spinal cord. Detailed assessments of a variety of behavioral tasks after a spinal cord injury and subsequent treatment can provide a first, "global" answer. In the case of the treatment with mAb IN-1, beneficial effects on the recovery of incomplete spinal cordinjured rats were reflected by an improved contact-placing response and normalized stride length during walking (Bregman et al., 1995). In the present study, numerous behavioral evaluations were achieved by using the well established BBB open field locomotor score (Basso et al., 1995), the grid walk test, and the narrow-beam test. The analysis was refined by the recording of muscle activity during treadmill walking, offering the possibility of transparently analyzing the locomotor pattern.

Our results show that $\mathrm{mAb} \mathrm{IN}-1$ treatment, which is known to enhance regeneration and plastic fiber growth, induces significant functional improvements in locomotion in the absence of changes in a sensory test (tail flick) in adult spinal cord-injured rats. 


\section{MATERIALS AND METHODS}

Animals. Experiments were performed on 43 adult Lewis rats of either sex (200-250 gm). All animals were analyzed behaviorally. Initially, an mAb IN-1-treated and a control antibody $(\mathrm{Ab})$ (anti-HRP) group $(n=$ 10 rats per group) were tested without the implantation of EMG electrodes. Another experimental set ( $n=10$ rats per group) was initiated, in which EMG electrodes were implanted $40 \mathrm{~d}$ after the injury. The implantation of EMG electrodes into uninjured rats was performed separately $(n=3)$.

All rats were kept in a $12 \mathrm{hr}$ light/dark cycle and received water and food ad libitum. The study was approved by the veterinary authorities of the Kanton of Zürich.

Surgical procedure. The animals were anesthetized with Dormicum [midazolam, $0.6 \mathrm{mg}$ per $100 \mathrm{gm}$ body weight (bw), i.p.; Roche, Basel, Switzerland] and Hypnorm (fentanyl, $0.02 \mathrm{mg}$ per $100 \mathrm{gm}$ bw, i.p.; Janssen-Cilag, Beerse, Belgium). To expose the spinal cord, a laminectomy of half a vertebra was performed at the thoracic level $\mathrm{Th}_{8}$. Using iridectomy scissors, a dorsal over-hemisection, sparing just parts of the ventral funiculus, was performed. Afterward, the dorsal musculature was sutured, and the skin was closed with surgical clips.

For constant antibody supply, hybridoma cells $\left(\sim 10^{6}\right)$ either producing mAb IN-1 (Caroni and Schwab, 1988) or anti-HRP antibodies (as a control) (Schnell and Schwab, 1990) were injected unilaterally into the hippocampal area. To prevent rejection of the hybridoma xenograft, the animals were immunosuppressed by daily injections of cyclosporin A (Sandimmun, $1.2 \mathrm{mg}$ per $100 \mathrm{gm}$ bw, i.p.; Novartis, Basel, Switzerland). The treatment commenced $1 \mathrm{~d}$ before surgery and continued for a total of $7 \mathrm{~d}$. For prophylactic reasons, doxycyclin (Vibravenoes, $0.85 \mathrm{mg}$ per $100 \mathrm{gm}$ bw, s.c.; Pfizer, Groton, CT) was injected once during the surgery. For postoperative pain relief, the animals received two applications (one every $24 \mathrm{hr}$ ) of rimadyl (Carprofen, $1 \mathrm{mg}$ per $100 \mathrm{gm}$ bw, s.c.; Pfizer). Until the rats displayed restored autonomic bladder function, the bladder-voiding reflex was triggered by a tender massage of the lower part of the abdomen three times a day. During the testing period, bladder infections occurred in two animals, which were then treated daily with antibiotics [cotrimoxazol (Bactrim), $2 \mathrm{mg}$ per $100 \mathrm{gm}$ bw, s.c.; Roche].

Behavioral testing. All tests were performed in a double-blind manner. Before the surgery, the animals were trained for 2 weeks before baseline measurements were taken. At $7 \mathrm{~d}$ after surgery, the testing sessions were performed in weekly intervals up to day 35 after surgery.

$B B B$ locomotor score. Open-field locomotion was evaluated by using the 21-point BBB locomotion scale (Basso et al., 1995). The rats were placed in an open field $(80 \times 130 \times 30 \mathrm{~cm})$ with a pasteboard-covered nonslippery floor. In each testing session, the animals were observed individually for $4 \mathrm{~min}$ by two observers. The hindlimb locomotion was then scored from 0 to 21 points (no observable locomotor movements to normal locomotor movements).

Grid walk. The animals had to walk on a 1-m-long horizontal runway of metal grid bars elevated $30 \mathrm{~cm}$ from the ground. A defined $10 \mathrm{bar}$ sector was chosen for analysis. To prevent habituation to a fixed bar distance, the bars in this sector were placed irregularly (1-4 cm spacing) and were changed in every testing session. Analysis was performed by counting the number of errors in foot placing; if the animal could not walk with its hindlimbs, it would make two errors per bar, resulting in a total of 20 errors.

Misplacement of a foot led to a withdrawal response, as described previously in cats (Gorassini et al., 1994; Hiebert et al., 1994). To calculate the retraction time after a stepping error, the rats were monitored with a digital video camera while crossing the grid. In the case of a stepping error of a hindlimb, the latency was measured from the beginning of the misstep (paw crossing a fictive ground line) (see Fig. $3 B$ ) until the animal started to retract the limb. At the end of the testing period, a minimum of five withdrawal movements per animal were recorded. In the baseline measurements, stepping mistakes were observed only very rarely. The average of the withdrawal latencies of those events was taken as a reference value.

Narrow-beam crossing. This paradigm assesses the ability of the rats to balance on $30 \mathrm{~cm}$ elevated wooden beams with a length of $1 \mathrm{~m}$. Different beam shapes were used to increase the level of difficulty: two beams with rectangular cross-sections $(2 \times 2 \mathrm{~cm} ; 1.2 \times 1.2 \mathrm{~cm})$ and a beam with a round cross-section (2.5 cm in diameter) (Metz et al., 1998). Crossing one beam by properly placing both hindlimbs was scored as 2 points; a total of 1.5 points was assigned when an animal placed only one paw plantar on the beam. Only 1 point was given if the rat was able to cross the whole beam but was unable to place the hindpaws, and 0.5 points was given if the rat could only traverse half of the beam. The score was zero in cases in which the rat was not able to cross at least half of the beam. The scores of all three beams were added to a maximum score of 6 points.

Electromyographic recordings. EMG recordings were acquired at postoperative day 40 in 10 lesioned and mAb IN-1-treated animals, 10 lesioned and control Ab-treated animals, and 3 unlesioned animals. Bipolar electrodes were implanted in each hindlimb into the vastus lateralis (VL) muscle (knee extensor) and the tibialis anterior (TA) muscle (ankle flexor). Under pentobarbital anesthesia (Nembutal, 50 $\mathrm{mg} / \mathrm{kg}$ bw, i.p.; Abbott, Irving, TX), the skull surface was exposed, and one self-tapering screw $(1.4 \times 7 \mathrm{~mm})$ was anchored on each side $2 \mathrm{~mm}$ lateral to the sagittal and $3 \mathrm{~mm}$ frontal to the lambdoidal suture of the skull. A 9-pin connector was fixed to the screws with dental acrylic cement (Paladur Cold-Curing). Teflon-insulated multistranded stainless steel wires (AS 632; Cooner Wire, Chatsworth, CA) were led from the connector subcutaneously via the back to the designated muscles. The wires were sutured into the muscles, whereby a $1 \mathrm{~mm}$ stripped region of each wire was placed within the muscle and served as an electrode. One wire was placed as a ground electrode subcutaneously on the back. Two days after electrode implantation, EMG recordings on the treadmill (running at a speed of $10.5 \mathrm{~m} / \mathrm{min}$ ) were performed. The headpiece on the rats was connected via a customized eight channel amplifier to an analog-to-digital board (Axoscope DigiData interface; Axon Instruments, Foster City, CA), and the signals were recorded with a sampling rate of $1 \mathrm{kHz}$ and filtered (high-pass, $30 \mathrm{~Hz}$; low-pass, $300 \mathrm{~Hz}$ ).

EMGs of $\sim 20$ step cycles were analyzed per rat (Axoscope; Axon Instruments). The step-cycle duration and burst duration of the TA and the VL muscles were measured. The deviation between step cycles was used as marker for rhythmicity. Uncoupling of the stepping pattern of the hindlimbs (irregular alternation between the muscle activity) (see Fig. $7 B$ ) was counted and set in relation to the number of performed steps $(n=20)$.

Tail flick. The level of nociception after spinal cord injury was evaluated by performing a standardized tail flick test (D'Amour and Smith, 1941; Gentsch et al., 1988). Rats were placed in a $17 \times 23 \mathrm{~cm}$ Plexiglas box and were first allowed to adjust to the new environment. When exploratory behavior ceased, an infrared source producing a calibrated heating beam (diameter, $1 \mathrm{~mm}$ ) was placed under the tail base and triggered together with a timer (Plantar tester, Ugo Basile Biological research apparatus; Ugo Basile, Comerio, Italy). The time for the first movement of the tail was noted. Each measurement was repeated three times, with at least a 4 min interval.

Lesion size (spared white matter). After the last testing session, the animals were deeply anesthetized with pentobarbital (Nembutal, 450 $\mathrm{mg} / \mathrm{kg}$ bw, i.p.) and perfused transcardially with a Ringer's solution containing 50,000 IU/1 heparin (Liquemin; Roche) and $0.25 \% \mathrm{NaNO}_{2}$, followed by a fixative solution (4\% paraformaldehyde solution in $0.1 \mathrm{M}$ phosphate buffer with $5 \%$ sucrose). The spinal cords were dissected, post-fixed overnight in the same fixative, and cryoprotected in a $30 \%$ sucrose solution for $3 \mathrm{~d}$. The tissue was then embedded in Tissue Tek (Satura Finetek, Torrance, CA) and frozen by immersion in $-40^{\circ} \mathrm{C}$ isopentane. Sagittal sections of $50 \mu \mathrm{m}$ were made on a cryostat and counterstained with cresyl violet.

The lesion size was measured with regard to its maximal rostrocaudal and its dorsoventral extension. Every second section was measured under a light microscope (Zeiss, Oberkochen, Germany) using 100× magnification.

The dorsoventral extension of ventral and dorsal white matter was measured in an uninjured cranial part of the spinal cord and taken as $100 \%$ white matter. The remaining white matter bridge at the epicenter of the lesion was then measured, and the ratio between the two measures was defined as a percentage of spared white matter (SWM). Using this method, every second section (16-20 per animal) was analyzed; from the SWM value of these sections, a mean value was calculated for each animal.

Statistics. Statistical comparisons were performed by using the MannWhitney $U$ test. $p$ values of $\leq 0.05$ were considered significant; values of $\leq 0.01$ were considered highly significant. Values represent means \pm SEM unless stated differently above in Electromyographic recordings. Regression lines were compared by using multiple regression analysis.

\section{RESULTS Lesion size}

In all control Ab-treated and mAb IN-1 Ab-treated rats, the lesion size was quantified. Thirty-four rats had a severe lesion 


\section{Control-Ab}
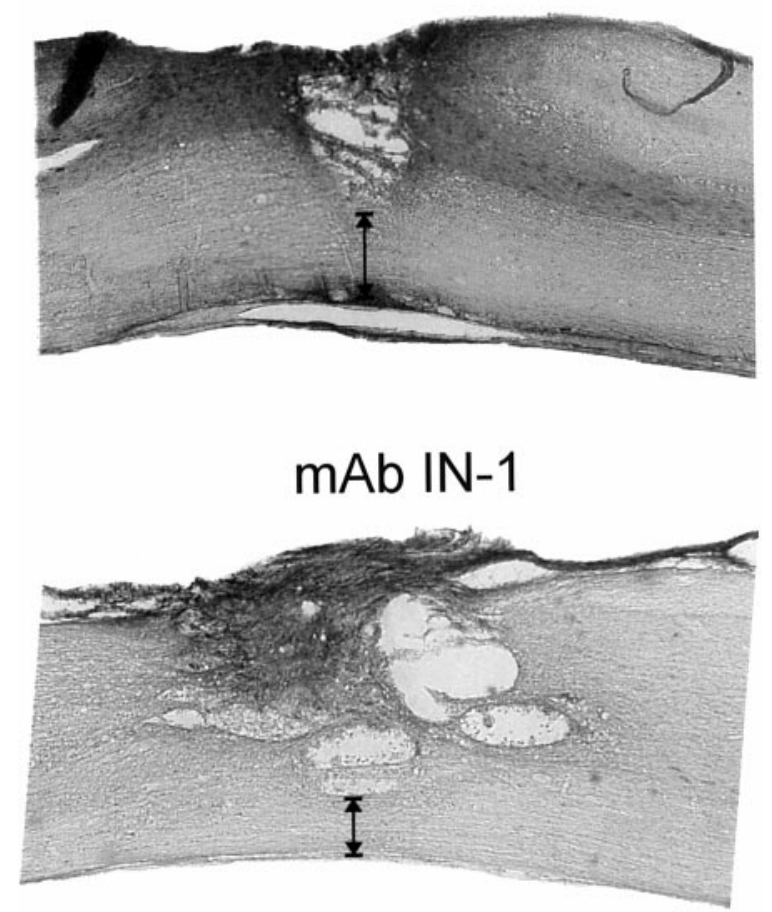

\section{$\overline{0.5 \mathrm{~mm}}$}

Figure 1. Evaluation of lesion size at the epicenter of the injury at thoracic level $\mathrm{Th}_{8}$. SWM was assessed from alternating sagittal sections of the spinal cord (arrows) and set in relation to the amount of white matter in the intact spinal cord rostral to the lesion (equaling 100\%). Sections at the center of the spinal cord are shown from a control animal and an IN-1 Ab-treated animal.

with complete destruction of the gray matter. Six animals with either a complete or too small lesion deviating more than twice the SD from the group mean were removed retrospectively from additional statistical evaluation. Three animals were left unlesioned for EMG analysis. In the remaining lesioned rats, the rostrocaudal extension of the lesion was between 1 and $2.75 \mathrm{~mm}$ with no significant difference between the control Ab-treated group and the mAb IN-1-treated group. A dense glial scar around the lesion site and the formation of cavities was observed in all of the animals (Fig. 1). Also the evaluation of the mean spared white matter showed no difference between both groups with $40.8 \pm$ $2.2 \%$ (mean \pm SEM) SWM in control Ab-treated rats and with $43.2 \pm 2.9 \% \mathrm{SWM}$ in mAb IN-1-treated rats.

All of the rats were analyzed for tumor remnants in the brain after perfusion. Enlarged ventricles, scar tissue, casts, and tumor debris were found in $80 \%$ of the animals (mAb IN-1 and control Ab groups).

\section{Open field locomotion (BBB score)}

The BBB locomotor score quantifies multiple aspects of spontaneous open-field, over-ground locomotion (Basso et al., 1995). The score reaches a maximum of 21 points, accomplished by all of the animals in the preoperative baseline measurements. Scores were very low and highly variable during the first 4-7 d after the injury, reflecting the spinal shock phase. Reproducible measurements could be taken from $7 \mathrm{~d}$ after lesion onward. Even at this early time point, a small difference between the two experimental groups could be observed: the BBB score in the control Abtreated group was $4.8 \pm 0.64(n=17)$, versus $6.1 \pm 0.83$ in the mAb IN-1-treated group ( $p>0.05 ; n=17)$ (Fig. $2 A)$. Four of the control Ab-treated rats but only two of the mAb IN-1-treated animals were not able to move their hindlimbs at this time point. These six animals exhibited a low amount of SWM $(<30 \%)$. The remaining animals were capable of moving one to three joints. Only three animals showed plantar placement of the paw; all of these animals were treated with mAb IN-1. Over the following 4 weeks, both groups showed substantial recovery of hindlimb movements (Fig. $2 A$ ). At day 35 after the injury, the mAb IN-1treated group reached a $\mathrm{BBB}$ score of $12.5 \pm 0.7$, which was significantly higher than that of the control Ab-treated group $(10.3 \pm 0.54$; Fig. $2 A)$. When comparing the single animals at this time point (Fig. $2 B$ ), the mAb IN-1-treated group could be split into two subgroups. Five of 17 animals failed to improve beyond a $\mathrm{BBB}$ level of 9, whereas 11 of 17 rats reached $\mathrm{BBB}$ scores of 12-17. In contrast, the control Ab-treated group appeared more homogenous, with minimum $\mathrm{BBB}$ scores of 8 and maximum scores of 14.

A strong correlation of the lesion size and the BBB score was observed in both groups, but the slope of the regression line in the mAb IN-1-treated rats was significantly steeper $(p=0.006)$, thereby indicating that the animals with a larger amount of spared white matter ( $>40 \%$ SWM) could benefit especially from the treatment (Fig. 2C).

\section{Grid walk}

The ability to precisely control and place the hindlimbs was tested on a horizontal, irregular ladder-like grid, as described in Materials and Methods. After a short preoperative training period, the animals were able to cross the grid almost without any faults (on average less than one mistake per crossing). Seven days after injury, rats of both groups were able to cross the grid but only by performing movements without accurate placing of the hindpaws, resulting in a high number of mistakes (Fig. $3 A$ ). After $14 \mathrm{~d}$, the control Ab-treated rats slightly improved to $14.6 \pm 1.1$ of a maximum of 20 mistakes. At the same time point, the average score of the mAb IN-1-treated group was significantly better $(10.7 \pm 0.9)$. Up to $35 \mathrm{~d}$ after the injury, the mAb IN-1-treated group further improved to $7.5 \pm 0.72$ mistakes, whereas the control Ab-treated animals made $10.8 \pm 1.1$ mistakes $(p=0.01)$ (Fig. 3A).

\section{Withdrawal response}

When an animal misses a grid bar, thus stepping into a hole, a withdrawal movement occurs, which is known to be modulated by supraspinal input (Hiebert et al., 1994). The delay from a step into a hole between two bars until the onset of the withdrawal movement of the hindlimb was determined as described in Materials and Methods (Fig. $3 B$ ). In the preoperative measurement, this latency was $170 \pm 6 \mathrm{msec}(n=29)$. At $35 \mathrm{~d}$ after lesion, 14 control Ab-treated rats and 15 mAb IN-1-treated animals showed a withdrawal response in the case of a misstep. In the control Ab group, the withdrawal latency was prolonged to $213 \pm 4 \mathrm{msec}$ (Fig. $3 C$ ). In contrast, the withdrawal latency of the mAb IN-1 group was not significantly different from the value of the intact animals, indicating a full recovery of this withdrawal response $(178 \pm 7 \mathrm{msec})$. 

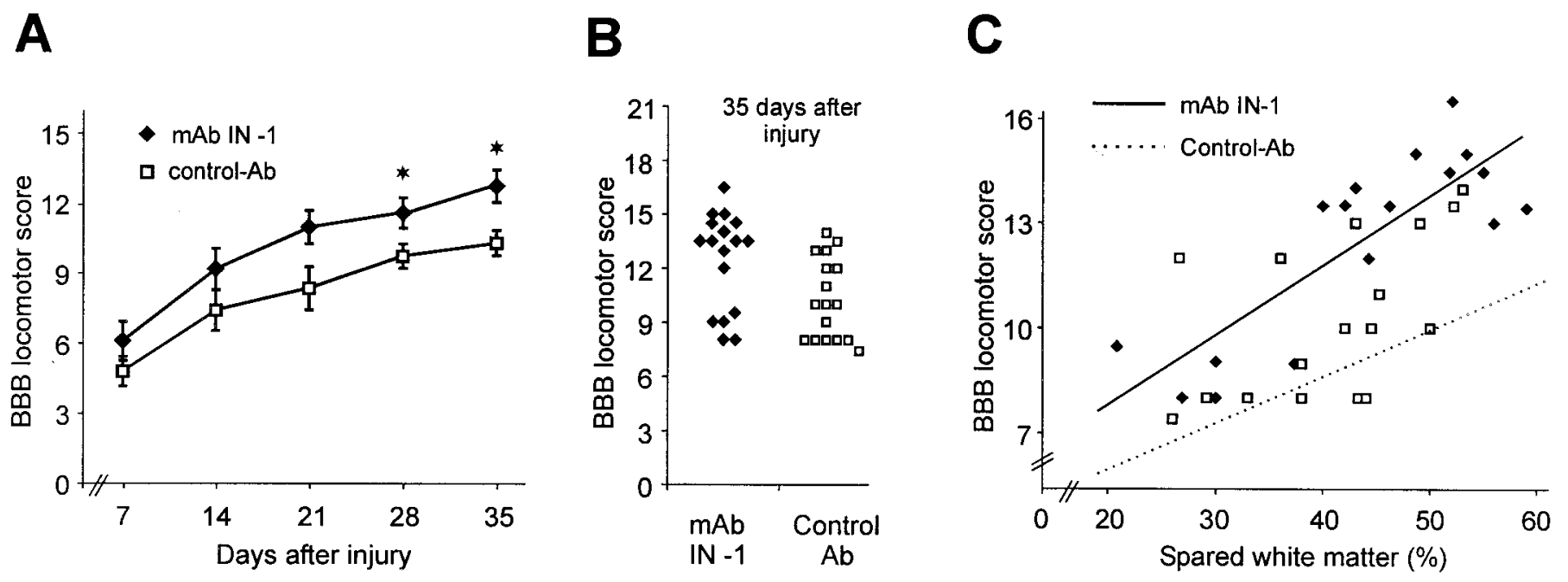

Figure 2. Recovery monitored with the BBB open-field locomotor score. A, Time course of the recovery in IN-1-treated and control Ab-treated rats $(n=17$ rats per group). $B$, Comparison of single animals with regard to BBB score $35 \mathrm{~d}$ after injury. Note the two subgroups in the mAb IN-1-treated animals. $C$, Correlation between SWM and the BBB locomotor score of single animals and the regression lines of the two groups. Data are given as means $\pm \mathrm{SEM} ;{ }^{*} p<0.05$.

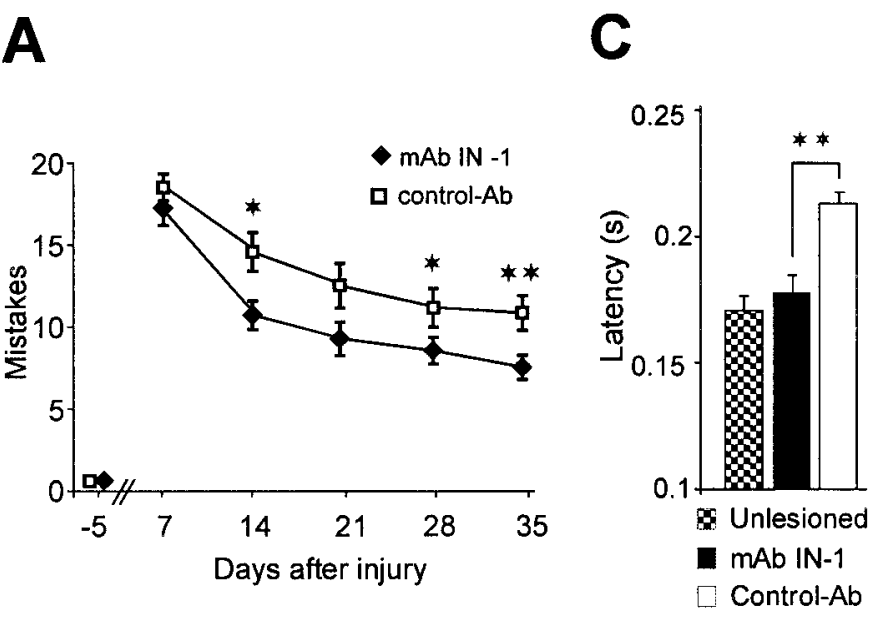

B

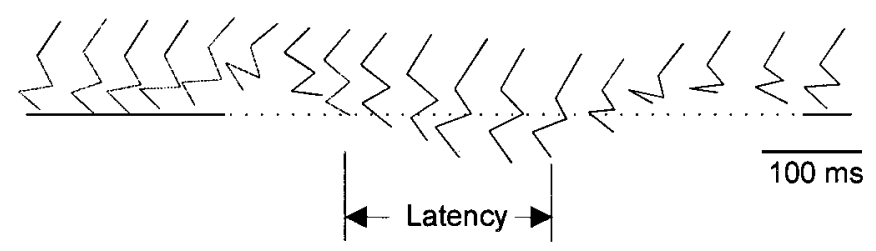

Figure 3. Grid walk performance. $A$, Time course of the recovery in IN-1-treated and control Ab-treated rats $(n=17$ rats per group) shows significantly lower error rates in the mAb IN-1 animals. $B$, Latency of the onset of the withdrawal movement in the case of a stepping error on the grid. The stick figure is illustrating the measurement (also see Materials and Methods). $C$, Bar graph (means \pm SEM) showing that mAb IN-1treated rats have recovered their response latency to preoperative values, in contrast to the control animals. ${ }^{*} p<0.05 ;{ }^{* *} p<0.01$.

\section{Narrow-beam crossing}

Before injury, all of the animals could cross the three differently shaped beams without any balancing difficulties (6 points) (Fig. 4). Seven days after the injury, the average narrow-beam score of the control Ab-treated rats was severely reduced from 6 to $0.3 \pm$
0.09 points, indicating that most rats lost balance as soon as they were placed on the beams. At this time point, the mAb IN-1treated rats showed an average score of $0.8 \pm 0.12$, reflecting their ability to cross the whole length of the broadest beam. Over the next few weeks, the performance in this test recovered modestly, so that at $35 \mathrm{~d}$ after the injury, the average narrow-beam score of the control $\mathrm{Ab}$ group was $1.2 \pm 0.17$ and that of the mAb IN-1-treated group was $2.1 \pm 0.25$. One mAb IN-1-treated rat was able to cross all three beams. Statistically, mAb IN-1 treatment led to a significant improvement as soon as $7 \mathrm{~d}$ after lesion $(p<0.05)$, which consistently increased during the whole testing period when compared with the control Ab-treated group. However, severe deficits were still obvious when compared with uninjured animals, probably because of the loss of proprioceptive information by the complete, bilateral dorsal column lesion.

\section{Electromyographic recordings during treadmill walking}

At the end of the second set of experiments (as described in Materials and Methods), EMG electrodes were implanted into the tibialis anterior and the vastus lateralis muscle of both hindlimbs in $10 \mathrm{mAb}$ IN-1-treated and 10 control Ab-treated rats. EMGs were recorded during stable treadmill walking of the rats. Evaluation of the EMGs revealed major and characteristic changes of the gait pattern in the spinal cord-injured animals (Fig. $5)$. In animals with a low BBB score $(<8)$, hardly any rhythmic extensor activity (i.e., VL muscle) could be observed (Fig. 5A, control Ab-treated animals). The flexor (i.e., TA muscle) was rhythmically active, but the burst duration was prolonged in comparison with uninjured animals (Fig. 5D, intact control animals), and the rhythm was fairly slow and irregular. Cocontractions between the flexor and the extensor muscles in one limb were detected frequently (see Fig. 7A). Because extensor activity was not patterned in some of the animals, no evaluation of this parameter could be performed. All of these parameters (i.e., rhythmic activity of flexor and extensor muscles, step-cycle duration, and rhythmicity) were improved in animals with higher locomotor scores, independent of their treatment (Fig. 5B, C, control Ab-treated and mAb IN-1-treated animals, respectively). This finding was confirmed by the correlation between the aver- 


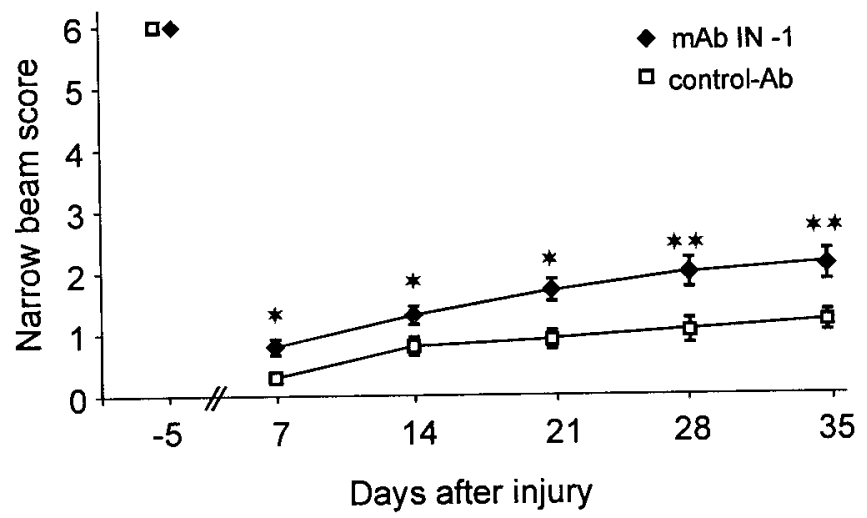

Figure 4. Time course of the recovery in IN-1-treated and control Ab-treated rats in the narrow-beam test ( $n=17$ rats per group). The ability of the rats to walk on differently shaped wooden beams is scored from 0 to 6 (see Materials and Methods). Data are given as means \pm $\mathrm{SEM} ;{ }^{*} p<0.05 ;{ }^{* *} p<0.01$.

age step-cycle duration or step-cycle variation of the individual animals (mAb IN-1-treated and control Ab-treated as well as unlesioned rats) with their corresponding individual $\mathrm{BBB}$ score (Fig. 6A,B). A correlation factor of $r=0.61$ and 0.65 was found for the duration and the variation (equaling the $\mathrm{SD}$ of step-cycle duration in single animals), respectively, indicating that both measurements are valuable indicators for the evaluation of the functional recovery. A comparison of both parameters between the treatment groups showed a strong recovery of stepping frequency and rhythmicity in mAb IN-1-treated animals (Fig. 6C). Whereas the difference between the mAb IN-1-treated group and the control Ab-treated group was highly significant for step-cycle duration and the variation, the mAb IN-1-treated group did not differ significantly from the preoperative values (normal animals). Measurements of the burst duration of the extensor muscle (possible in seven control Ab-treated rats and eight mAb IN-1-treated rats) showed a significant increase in both the mAb IN-1-treated group and the control Ab-treated group compared with unlesioned animals. There was no statistical difference between the two treatment groups $(285.7 \pm 20.4$ in unlesioned rats, $461.9 \pm 42$ in $\mathrm{mAb}$ IN-1-treated rats, and $505.1 \pm 55.5$ in control Ab-treated rats). The flexor burst duration was modestly increased only in the control Ab-treated group, but this increase was not statistically significant (139.1 \pm 5.6 in unlesioned rats, $146.4 \pm 9.8$ in $\mathrm{mAb}$ IN-1-treated rats, and $236.4 \pm 80.3$ in control Ab-treated rats).

Uncoupling of the two hindlimbs (Fig. 7B) was observed in the spinal cord-injured rats of both treatment groups (mAb IN-1 and control $\mathrm{Ab}$ ), but never in the unlesioned animals. When such uncoupling events were compared (during 20 steps) between the two treatment groups, the mAb IN-1-treated rats performed significantly better $(1.5 \pm 0.7$ uncoupling events per 20 steps $)$ than the control Ab-treated rats $(3.3 \pm 0.35$ events $)(p<0.05)$.

\section{Tail flick}

Uncontrolled fiber growth in the spinal cord could lead to malfunctions such as neurogenic pain. We used the tail flick test to assess changes in this single spinal reflex pathway. The delay from the beginning of heating the tail base to the first withdrawal movement of the tail in preoperative baseline measurements was $5.0 \pm 0.9 \mathrm{sec}$ for the future mAb IN-1-treated group and $4.5 \pm 0.5$ sec for the future control Ab-treated group ( $n=17$ rats per group) (Fig. 8). At 5 weeks after the lesion, this withdrawal
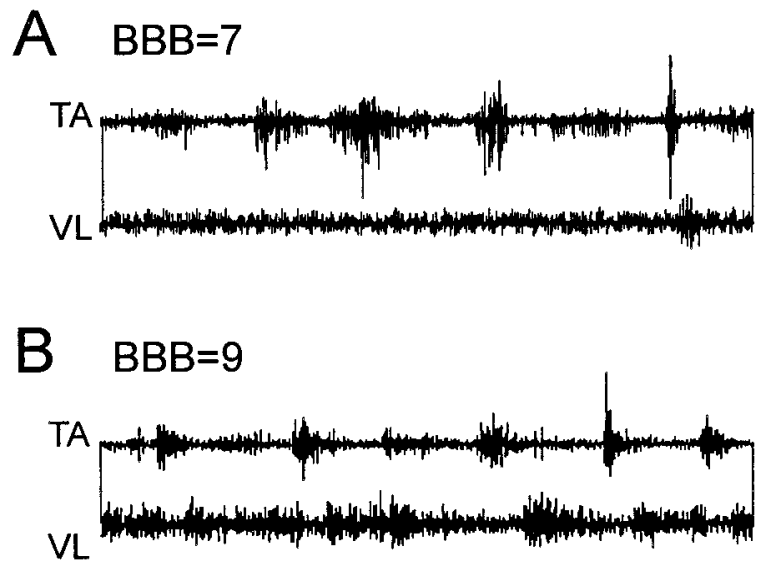

C $\mathrm{BBB}=15$
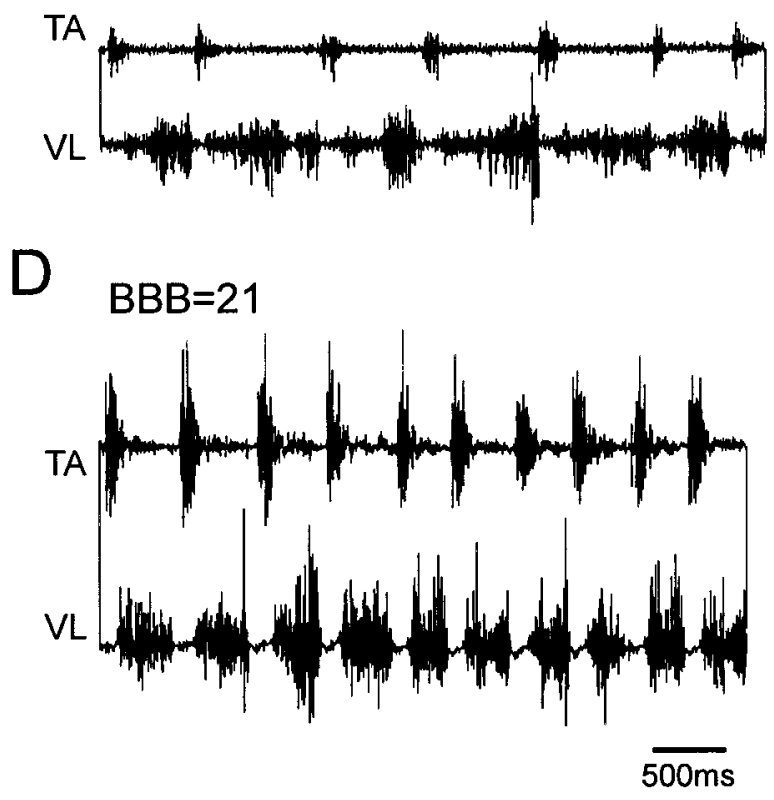

Figure 5. EMG recordings of treadmill-walking rats $40 \mathrm{~d}$ after lesion from two hindlimb muscles in rats with different locomotor scores. $A$, Only the recorded flexor muscle TA was found to be rhythmically active. In comparison with an uninjured rat $(D)$, the contractions were prolonged and irregular, and the frequency was fairly low. The extensor muscle VL rarely shows activity. $B$, The extensor muscle $\mathrm{VL}$ is more active and bursts in a more rhythmic pattern. Cocontractions between the muscles occurred frequently. $C$, The flexor $(T A)$ rhythm is increased, and the bursts are more defined and shorter. In addition, the extensor $(V L)$ bursts are more regular and are well coordinated with the flexor muscle. $D$, Recordings of an intact control animal.

movement could still be consistently initiated in all of the animals. Its latency was very similar to the baseline measurements: the withdrawal time was $5.3 \pm 1.7 \mathrm{sec}$ in the mAb IN-1-treated group and $5.0 \pm 1.2 \mathrm{sec}$ in the control $\mathrm{Ab}$ group, with no significant difference between both groups (Fig. 8).

\section{DISCUSSION}

In this study, we examined whether the enhanced anatomical reorganization of the adult CNS occurring after lesion and the 

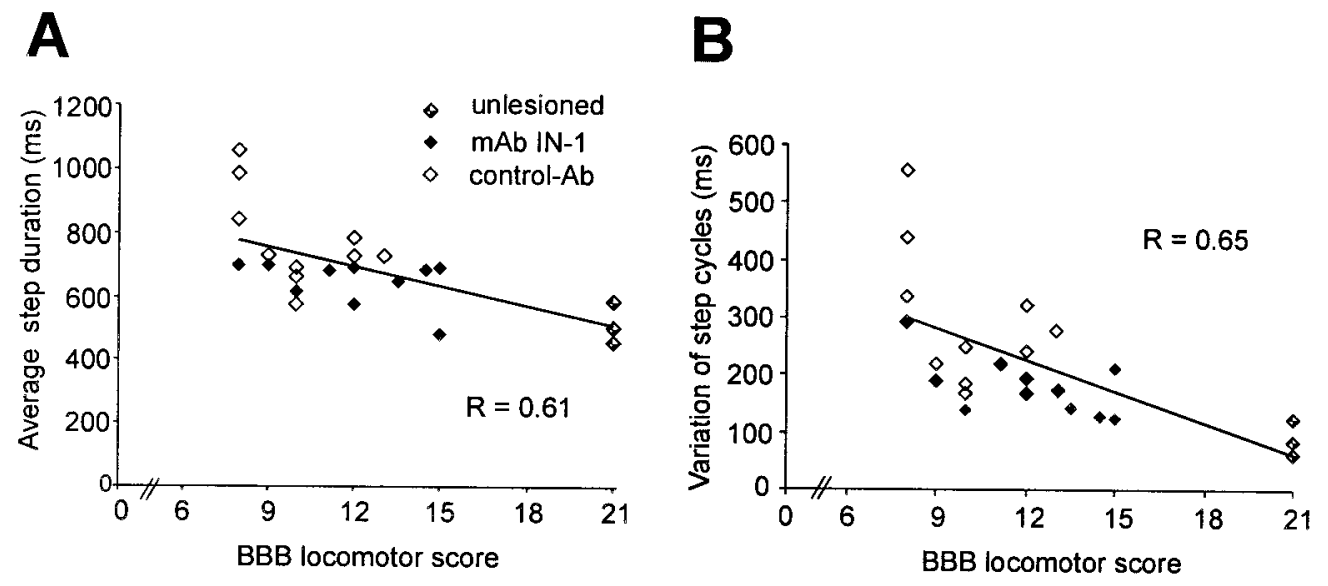

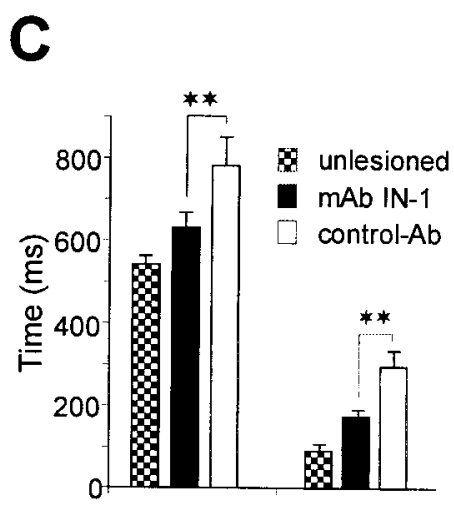

Average step Variation of step duration (ms) cycles (ms)

Figure 6. Evaluation of EMG recordings $40 \mathrm{~d}$ after injury: step-cycle duration and rhythmicity. A, The correlation between the BBB locomotor score and the step-cycle duration of all EMG implanted rats $40 \mathrm{~d}$ after lesion showed a decrease in the duration with increasing performance in the open field. $B$, A correlation between the deviation in the step-cycle duration and the BBB locomotor score $40 \mathrm{~d}$ after lesion indicates a strong relationship between an increase in open field performance and a more regular stepping pattern. $C$, A comparison of the average step-cycle duration (means \pm SEM) as well as its variation (shown as SD) between IN-1-treated and control Ab-treated rats shows a highly significant improvement in the mAb IN-1 group. ${ }^{* *} p<0.01$.

treatment with the Nogo-A-neutralizing antibody mAb IN-1 (Schnell and Schwab, 1990, 1993; Bregman et al., 1995; Thallmair et al., 1998; Raineteau et al., 1999; Brosamle et al., 2000; Buffo et al., 2000; Chen et al., 2000) is beneficial for the locomotor recovery after a severe spinal cord injury. We found that all animals recovered substantially from their injury, but the mAb IN-1-treated rats performed in all applied locomotor tests significantly better than the control Ab-treated rats. Recordings of muscle EMG activity during treadmill walking confirmed these results and showed clear differences between the two treatment groups in step-cycle duration, rhythmicity, and limb coordination. In these parameters, the mAb IN-1-treated group showed values not significantly different from unlesioned animals. The tail withdrawal reflex that occurred during thermal stimulation was unchanged in both treatment groups, without a difference between the control and mAb IN-1-treated group, indicating the absence of an increased pain threshold.

Our results are consistent with earlier findings in that $\mathrm{mAb}$ IN-1 treatment after injuries of the adult CNS leads to functional benefits (Bregman et al., 1995; Thallmair et al., 1998). The relatively high number of animals used in our study allows a clear statistical interpretation of the data, despite considerable variation in lesion size and outcome typical for spinal cord lesion experiments (Noble and Wrathall, 1989; Basso et al., 1996). The preservation of a few ventrolateral fibers can result in large differences in locomotor performance (Rossignol, 1996; Brustein and Rossignol, 1998). Histological evaluation showed that the ventrally located reticulospinal and vestibulospinal tracts were at least partly intact in all injured rats (data not shown). These tracts are important for the initiation and the control of the locomotor pattern (Brustein and Rossignol, 1998; Jordan, 1998), which is generated by spinal pattern-generating networks (Grillner, 1985; Pearson, 1993). This probably explains our finding that the animals of both groups were able to initiate a rhythmic movement pattern of the hindlimbs and to locomote on a flat surface as demonstrated in the BBB locomotor score. Because in the BBB score points are not assigned in a linear manner, functional importance of single points for the recovery of stepping should not be expressed by using absolute values only. In our study, the control Ab-treated rats reached a mean of 10 points at $35 \mathrm{~d}$ after injury, representing occasional $(<50 \%)$ weight-supported plantar steps with no forelimb-hindlimb coordination. In contrast, the $\mathrm{mAb}$ IN-1-treated rats reached a mean of 12.5 points (good weight support and nearly consistent forelimb-hindlimb coordination), indicating major improvements in the recovery of walking.

In contrast to the ventral tracts, the dorsal and dorsolateral corticospinal tract (CST) and rubrospinal tract were completely disconnected in all of the animals. These tracts are mainly involved in fine control of movements (Armstrong, 1986; Kennedy, 1990; Whishaw et al., 1992), and the corticospinal tract has been shown to be relatively unimportant for plain locomotion (Metz et al., 1998, Muir and Whishaw, 1999). This was reflected by severe deficits in those tests requiring precise motor control (i.e., in the grid walk or in the narrow-beam test). Although there were still severe deficits present, the $\mathrm{mAb}$ IN-1-treated rats recovered significantly better in these tests, possibly reflecting a partial reestablishment of descending control in these animals. As another test for descending control, we evaluated the latency of the withdrawal response in the case of a misstep on the grid. Supraspinal input facilitates the activation of this spinal reflex, as shown in spinalized (complete spinal-cord injured) cats in which the withdrawal response elicited by loss of ground was delayed compared with intact animals (Gorassini et al., 1994; Hiebert et al., 1994). Similar to the results obtained with cats, our results show that the initiation of the withdrawal response was delayed after spinal cord injury also in rats. However, this has been found only in control Ab-treated animals, whereas the mAb IN-1treated group had recovered to the preoperative level $35 \mathrm{~d}$ after injury.

The functional recovery observed here probably involves multiple plastic and regenerative changes on different levels of the motor system. On the cortical level, adaptive changes of the motor map have been observed in spinal cord-injured humans and animals (Bruehlmeier et al., 1998) as well after limb amputation (Kaas et al., 1999).

Plastic modification also seem to occur on the level of descending spinal tracts, because spontaneous recovery of locomotion 
A

Co-contractions

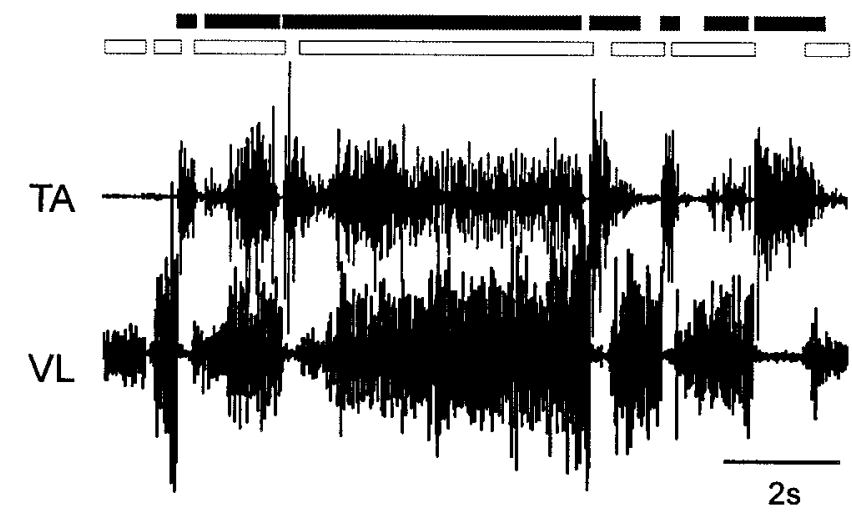

B

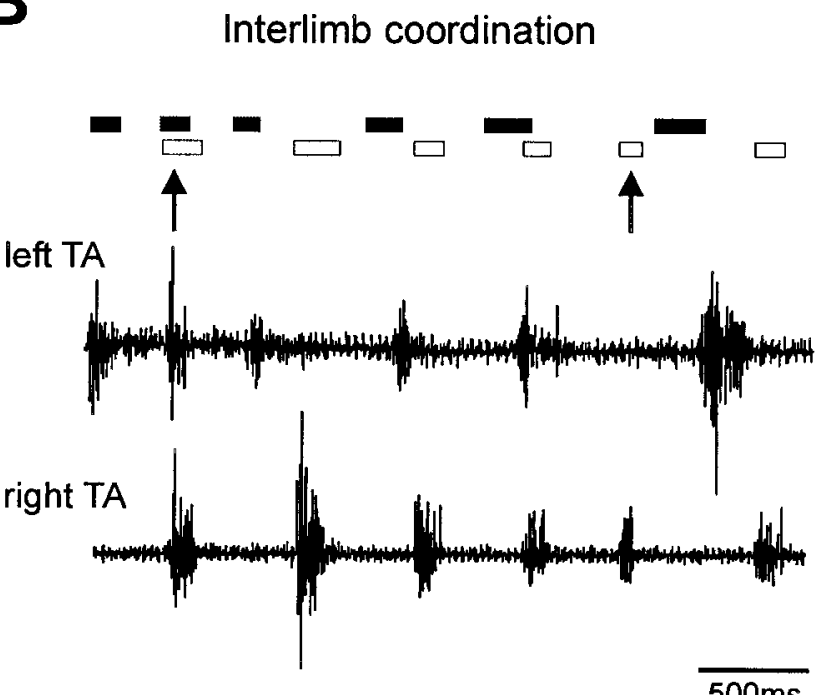

Figure 7. $A$, Cocontractions between the VL and the TA occurred frequently in all spinal cord-injured rats especially at BBB scores of $<12$. Because some animals did not show patterned extensor activity, no statistical comparison was performed. The black bars and white bars represent the activity pattern of the TA and the VL muscles, respectively. $B$, Uncoupling of the rhythmically active hindlimbs (arrows) occurred in animals of both treatment groups, especially in rats with a BBB score of $<10$. An example for the right and left TA is shown.

after incomplete lesions was observed in cats and humans. Even very small amounts of remaining ventrolateral white matter were sufficient to initiate locomotion (Windle et al., 1958; Nathan, 1994). At the level of the spinal central pattern-generating network, activity-dependent modifications were recently described (De Leon et al., 1999; Pearson et al., 1999).

Because the mAb IN-1-treated rats improved significantly better than the control Ab-treated animals, enhancement of spontaneous plasticity and/or additional effects have to be taken into account. Regeneration of injured CST fibers occurs in the lesion paradigm used here after mAb IN-1 treatment (Schnell and Schwab, 1990, 1993; Bregman et al., 1995; Brosamle et al., 2000) and may contribute to the observed recovery. Furthermore, it has been shown that the mAb IN-1 allows sprouting and reorganization of lesioned as well as unlesioned fibers in adult rats at a degree that is normally only observed after perinatal lesions

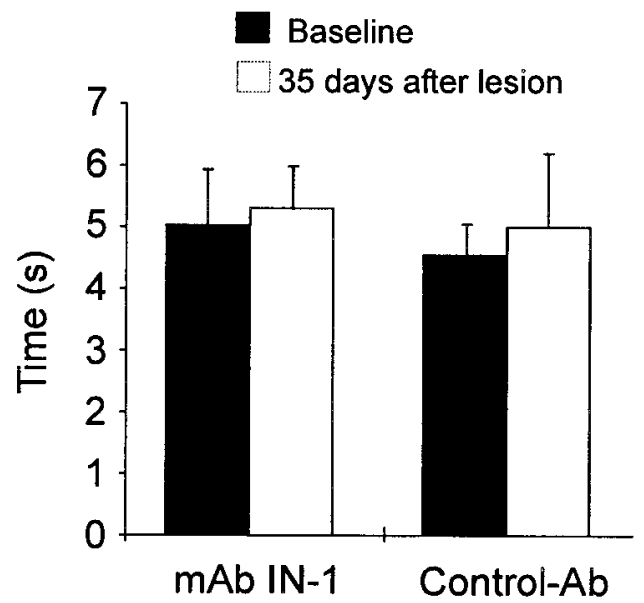

Figure 8. No significant changes were observed in the withdrawal response to a nociceptive stimulus (infrared beam) at $35 \mathrm{~d}$ after injury in the tail flick test (means $\pm \mathrm{SEM} ; n=17$ rats per group).

(Thallmair et al., 1998; Z'Graggen et al., 1998, 2000). Recent results have shown that after mAb IN-1 treatment even the rubrospinal tract can sprout and functionally innervate CST targets after CST removal (O. Raineteau, K. Fouad, P. Noth, and M. E. Schwab, unpublished observation). In the intact adult cerebellum, antibodies neutralizing Nogo-A induce transient sprouting of Purkinje cell axons (Buffo et al., 2000). This sprouting occurs as early as $2 \mathrm{~d}$ after antibody injection. Such plastic rearrangements could be the basis of the fast recovery observed in the present study.

Using our histological analysis approach of spared white matter, we found that only animals with higher amounts of SWM $(>40 \%)$ (Fig. $2 C$ ) benefited in the BBB locomotor score from the mAb IN-1 treatment. This result indicates that for functionally meaningful plastic rearrangements to occur, a minimal amount of preserved descending input is necessary and/or regenerating fibers depend on a minimum bridge size as a growth substrate. One has to keep in mind that by using the method described, small but critical differences in the lesion size could be missed, which might explain the deviation in the results (Fig. $2 C$ ).

Another important feature of the mAb IN-1-enhanced recovery is the fast onset, starting already at $7 \mathrm{~d}$ after lesion. Although little information on antibody concentration and spatial distribution after hybridoma cell implantation is available, we believe that the fast recovery is mediated by the IN-1 antibodies. The application method via antibody-producing hybridoma cells involves a certain variability; however, we found a detectable amount of antibodies in the blood serum of treated animals already at $4 \mathrm{~d}$ after implantation (our unpublished data). The recovery described in this study parallels the rapidly observed improvements in forelimb food pellet-grasping tests after lesions of the pyramidal tract and subsequent mAb IN-1 treatment (Thallmair et al., 1998; Z'Graggen et al., 1998). An earlier testing session has not been considered because the results would be misleading as a result of the spinal shock phase, which lasts for several days and varies from animal to animal (Holaday and Faden, 1983; Basso et al., 1996). Regenerating fibers can grow $>1 \mathrm{~mm} / \mathrm{d}$ in the rat spinal cord (Schnell and Schwab, 1990; Li and Raisman, 1994), and sprouting occurs rapidly (2-5 d) after mAb IN-1 treatment (Buffo et al., 2000). 
Because of the Ab delivery strategy (via hybridoma cells used), the time of IN-1 antibody supply was limited to $\sim 10 \mathrm{~d}$; nevertheless, the effects on the functional outcome are encouraging. The observed functional recovery indicates that the mAb IN-1 treatment leads to a meaningful rewiring of circuitry in the lesioned CNS. Cues and mechanisms for synapse formation, for integration of newly grown fibers into existing circuits, and for stabilization of connections still appear to be present or to be reexpressed as a consequence of the lesion in the adult rat CNS.

Enhanced fiber growth because of a treatment may also include a certain danger. A possible example could be neuropathic pain (e.g., after incomplete spinal cord injury) (Eide, 1998). The mechanisms causing neuropathic pain after spinal cord lesion are to a large extent unclear, but inappropriate sprouting of afferent fibers has often been considered (Woolf and Doubell, 1994; Christensen and Hulsebosch, 1997; Kennedy et al., 1997). Although so far such growth of sensory fibers has not been examined, it was of special interest to study whether the mAb IN-1 treatment influences pain perception or leads to hyper-reflexia. According to the classical tail flick test, spinal cord injury did not cause a change in reaction time, and no difference was observed between the IN-1-treated group and the control Abtreated group. This suggests either that sprouting did not occur or that no inappropriate connections of afferent fibers have been formed.

In conclusion, the present study shows that the mAb IN-1 treatment enhances functional recovery after spinal cord lesions, probably by allowing the formation of functionally meaningful new connections of uninjured and also severed axons in the adult rat CNS.

\section{REFERENCES}

Armstrong DM (1986) Supraspinal contribution to the initiation and control of locomotion in the cat. Prog Neurobiol 26:273-361.

Basso DM, Beattie MS, Bresnahan JC (1995) A sensitive and reliable locomotor rating scale for open field testing in rats. J Neurotrauma 12:1-21.

Basso DM, Beattie MS, Bresnahan JC (1996) Graded histological and locomotor outcomes after spinal cord contusion using the NYU weightdrop device versus transection. Exp Neurol 139:244-256.

Bregman BS, Kunkel-Bagden E, Schnell L, Dai HN, Gao D, Schwab ME (1995) Recovery from spinal cord injury mediated by antibodies to neurite growth inhibitors. Nature 378:498-501.

Brosamle C, Huber AB, Fiedler M, Skerra A, Schwab ME (2000) Regeneration of lesioned corticospinal tract fibers in the adult rat induced by a recombinant, humanized IN-1 antibody fragment. J Neurosci 20:8061-8068.

Bruehlmeier M, Dietz V, Leenders KL, Roelcke U, Missimer J, Curt A (1998) How does the human brain deal with a spinal cord injury? Eur J Neurosci 10:3918-3922.

Brustein E, Rossignol S (1998) Recovery of locomotion after ventral and ventrolateral spinal lesions in the cat. I. Deficits and adaptive mechanisms. J Neurophysiol 80:1245-1267.

Buffo A, Zagrebelsky M, Huber AB, Skerra A, Schwab ME, Strata P, Rossi F (2000) Application of neutralizing antibodies against NI-35/ 250 myelin-associated neurite growth inhibitory proteins to the adult rat cerebellum induces sprouting of uninjured Purkinje cell axons. J Neurosci 20:2275-2286.

Caroni P, Schwab ME (1988) Two membrane protein fractions from rat central myelin with inhibitory properties for neurite growth and fibroblast spreading. J Cell Biol 106:1281-1288.

Chen MS, Huber AB, van der Haar M, Frank M, Schnell L, Spillmann AA, Christ F, Schwab ME (2000) Nogo-A is a myelin-associated neurite outgrowth inhibitor and an antigen for monoclonal antibody IN-1. Nature 403:434-439.

Cheng H, Cao Y, Olson L (1996) Spinal cord repair in adult paraplegic rats: partial restoration of hind limb function. Science 272:510-513.

Christensen MD, Hulsebosch CE (1997) Chronic central pain after spinal cord injury. J Neurotrauma 14:517-537.

D'Amour FF, Smith GL (1941) A method for determining loss of pain sensation. J Pharmacol Exp Ther 72:74-79.

De Leon RD, Tamaki H, Hodgson JA, Roy RR, Edgerton VR (1999)
Hindlimb locomotor and postural training modulates glycinergic inhibition in the spinal cord of the adult spinal cat. J Neurophysiol $82: 359-369$.

Eide PK (1998) Pathopysiological mechanisms of central neuropathic pain after spinal cord injury. Spinal Cord 36:601-612.

Franklin RJM, Barnett SC (2000) Olfactory ensheathing cells and CNS regeneration: the sweet smell of success? Neuron 28:15-18.

Gentsch C, Lichtensteiner M, Frischknecht HR, Feer H, Siegfried B (1988) Isolation-induced locomotor hyperactivity and hypoalgesia in rats are prevented by handling and reversed by resocialisation. Physiol Behav 43:13-16.

Gorassini MA, Prochazka A, Hiebert GW, Gauthier MJ (1994) Corrective responses to loss of ground support during walking. I. Intact cats. J Neurophysiol 71:603-610.

Grill R, Murai K, Blesch A, Gage FH, Tuszynski MH (1997) Cellular delivery of Neutrophin-3 promotes corticospinal axonal growth and partial functional recovery after spinal cord injury. J Neurosci $17: 5560-5572$.

Grillner S (1985) Neurobiological base of rhythmic motor acts in vertebrates. Science 228:143-149.

Guénard V, Xu XM, Bunge MB (1993) The use of Schwann cell transplantation to foster central nervous system repair. Semin Neurosci 5:401-411.

Hiebert GW, Gorassini MA, Jiang W, Prochazka A, Pearson KG (1994) Corrective responses to loss of ground support during walking. II. Comparison of intact and chronic spinal cats. J Neurophysiol 71:611-622.

Holaday JW, Faden AI (1983) Spinal shock and injury: experimental therapeutic approaches. Adv Shock Res 10:95-98.

Jordan LM (1998) Initiation of locomotion in mammals. Ann NY Acad Sci 860:83-93.

Kaas JH, Florence SL, Jain N (1999) Subcortical contributions to massive cortical reorganisation. Neuron 22:657-660.

Kennedy PR (1990) Corticospinal, rubrospinal, and rubro-olivary projections: a unifying hypothesis. Trends Neurosci 13:474-479.

Kennedy P, Frankel H, Gardner B, Nuseibeh I (1997) Factors associated with acute and chronic pain following traumatic spinal cord injuries. Spinal Cord 35:814-817.

Kobayashi NR, Fan DP, Giehl KM, Bedard AM, Wiegand SJ, Tetzlaff W (1997) BDNF and NT-4/5 prevent atrophy of rat rubrospinal neurons after cervical axotomy, stimulate GAP-43 and T $\alpha 1$-tubulin mRNA expression, and promote axonal regeneration. J Neurosci 17:9583-9595.

Li Y, Raisman G (1994) Schwann cells induce sprouting in motor and sensory axons in the adult rat spinal cord. J Neurosci 14:4050-4063.

Li Y, Field PM, Raisman G (1997) Repair of adult rat corticospinal tract by transplants of olfactory ensheathing cells. Science 277:2000-2002.

Liu Y, Kim D, Himes BT, Chow SY, Schallert T, Murray M, Tessler A, Fischer I (1999) Transplants of fibroblasts genetically modified to express BDNF promote regeneration of adult rat rubrospinal axons and recovery of forelimb function. J Neurosci 19:4370-4387.

Metz GAS, Dietz V, Schwab ME, van de Meent H (1998) The effects of unilateral pyramidal tract section on hindlimb motor performance in the rat. Behav Brain Res 96:37-46.

Muir GD, Whishaw IQ (1999) Complete locomotor recovery following corticospinal tract lesions: measurement of ground reaction forces during overground locomotion in rats. Behav Brain Res 103:45-53.

Nathan PW (1994) Effects on movements of surgical incisions into the human spinal cord. Brain 117:337-346.

Noble LJ, Wrathall JR (1989) Correlative analyses of lesion development and functional status after graded spinal cord contusive injuries in the rat. Exp Neurol 103:34-40.

Pearson KG (1993) Common principles of motor control in vertebrates and invertebrates. Annu Rev Neurosci 16:265-297.

Pearson KG, Fouad K, Misiaszek JE (1999) Adaptive changes in motor activity associated with functional recovery following muscle denervation in walking cats. J Neurophysiol 82:370-381.

Raineteau O, Z'Graggen WJ, Thallmair M, Schwab ME (1999) Sprouting and regeneration after pyramidotomy and blockade of the myelinassociated neurite growth inhibitors NI 35/250 in adult rats. Eur J Neurosci 11:1486-1490.

Ramer MS, Priestley JV, McMahon SB (2000) Functional regeneration of sensory axons into the adult spinal cord. Nature 403:312-316.

Ramon-Cueto A, Cordero MI, Santos-Benito FF, Avila J (2000) Functional recovery of paraplegic rats and motor axon regeneration in their spinal cords by olfactory ensheathing glia. Neuron 25:425-435.

Rossignol S (1996) Neural control of stereotypic limb movements. In: Exercise: regulation and integration of multiple systems, Handbook of physiology, Sec 12 (Rowell LB, Sheperd JT, eds), pp 173-216. Oxford: American Physiologic.

Schnell L, Schwab ME (1990) Axonal regeneration in the rat spinal cord produced by an antibody against myelin-associated neurite growth inhibitors. Nature 343:269-272.

Schnell L, Schwab ME (1993) Sprouting and regeneration of lesioned corticospinal tract fibers in the adult rat spinal cord. Eur J Neurosci 5:1156-1171. 
Schnell L, Schneider R, Kolbeck R, Barde YA, Schwab ME (1994) Neurotrophin-3 enhances sprouting of corticospinal tract during development and after adult spinal cord lesion. Nature 367:170-173.

Thallmair M, Metz GAS, Z'Graggen WJ, Raineteau O, Kartje GL, Schwab ME (1998) Neurite growth inhibitors restrict plasticity and functional recovery following corticospinal tract lesions. Nat Neurosci $1: 124-131$.

Whishaw IQ, Pellis SM, Pellis VC (1992) A behavioral study of the contributions of cells and fibers of passage in the red nucleus of the rat to postural righting, skilled movements, and learning. Behav Brain Res 52:29-44.

Windle WF, Smart JO, Beers JJ (1958) Residual function after subtotal spinal cord transection in adult cats. Neurology 8:518-521.
Woolf CJ, Doubell TP (1994) The pathophysiology of chronic pain: increased sensitivity to low threshold A $\beta$-fibre inputs. Curr Opin Neurobiol 4:525-534.

Xu XM, Guenard V, Kleitman N, Bunge MB (1995) Axonal regeneration into Schwann cell-seeded guidance channels grafted into transected adult rat spinal cord. J Comp Neurol 351:145-160.

Z'Graggen WJ, Metz GAS, Kartje GL, Thallmair M, Schwab ME (1998) Functional recovery and enhanced corticofugal plasticity after unilateral pyramidal tract lesion and blockade of myelin-associated neurite growth inhibitors in adult rats. J Neurosci 18:4744-4757.

Z'Graggen WJ, Fouad K, Raineteau O, Metz GA, Schwab ME, Kartje GL (2000) Compensatory sprouting and impulse rerouting after unilateral pyramidal tract lesion in neonatal rats. J Neurosci 20:6561-6569. 